JGR Oceans

\author{
RESEARCH ARTICLE \\ 10.1029/2019JC015676 \\ Key Points: \\ - A new method using sigma-pi \\ diagram to classify water masses is \\ proposed and applied to the \\ northern South China Sea \\ - Thirteen water masses in the \\ northern South China Sea are \\ classified with the new method \\ - The westward intrusion of the West \\ Pacific Ocean water can be studied \\ by potential spicity analysis
}

Correspondence to:

J. $\mathrm{Hu}$,

hujy@xmu.edu.cn

Citation:

Gao, Y., Huang, R. X., Zhu, J., Huang, Y., \& Hu, J. (2020). Using the sigma-pi diagram to analyze water masses in the northern South China Sea in spring. Journal of Geophysical Research: Oceans, 125, e2019JC015676. https:// doi.org/10.1029/2019JC015676

Received 20 SEP 2019

Accepted 17 JUN 2020

Accepted article online 20 JUN 2020

(C)2020. American Geophysical Union. All Rights Reserved.

\section{Using the Sigma-Pi Diagram to Analyze Water Masses in the Northern South China Sea in Spring}

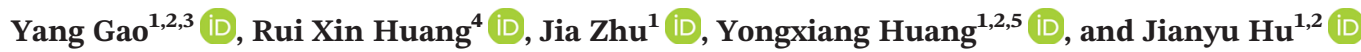 \\ ${ }^{1}$ State Key Laboratory of Marine Environmental Science, College of Ocean and Earth Sciences, Xiamen University, \\ Xiamen, China, ${ }^{2}$ Southern Marine Science and Engineering Guangdong Laboratory, Zhuhai, China, ${ }^{3} \mathrm{CNRS}$, Univ. Lille, \\ Univ. Littoral Cote d'Opale, UMR 8187, LOG, Laboratoire d'Océanologie et de Géosciences, Wimereux, France, \\ ${ }^{4}$ Department of Physical Oceanography, Woods Hole Oceanographic Institution, Woods Hole, MA, USA, \\ ${ }^{5}$ Fujian Engineering Research Center for Ocean Remote Sensing Big Data, Xiamen, China
}

\section{Introduction}

Water mass can be treated as a huge bulk of water body formed in a specific region with unique biophysical-chemical features that are continuously distributed and in a long-term stable state. However, the water mass still exhibits spatial and temporal variations due to mixing and changes in the environment, including climate variability, which can greatly affect the oceanic state, climate, and navigation, to list a few. Besides, the boundaries of water masses are critical to study large-scale thermohaline circulation that is widely recognized to play a crucial role in global climate change and, thus, Earth systems (Thorpe, 2005). In oceanic studies, water mass analysis is vitally important. The most widely used method in water mass analysis is based on the temperature-salinity (T-S) diagram, by which water masses are classified according to their temperature and salinity properties. Even though one can roughly locate the water mass center using the original or improved T-S diagram (Li et al., 2004), the boundaries between these water masses are poorly defined.

Apart from the traditional T-S diagrams, several methods have been proposed in the field of water mass classification. For example, Qiu et al. (1984) used the stepwise cluster method to analyze the water masses in the Huanghai Sea. Su et al. (1989) applied the cluster analysis method to classify the modified water masses in the Huanghai Sea and East China Sea. Chen and Du (1990) classified the upper layer water in the Equatorial Western Pacific using the iterative self-organizing data analysis technique of the fuzzy cluster analysis. Jan et al. (2010) classified the water masses in the Taiwan Strait using cluster analysis and the associated T-S diagrams. In these statistical cluster analysis methods, the data set is classified into groups according to the similarity in parameters among data. In addition, the similarity is often estimated by the squared Euclidean distance based on the normalized $\mathrm{T}$ and $\mathrm{S}$ differences between points. To improve the practicability of fuzzy cluster analysis, Zhu et al. (2019) introduced the T-S similarity number (TSSN) to further quantify the water sample by specifying the boundaries between the water masses. Apart from the T-S-based or fuzzy cluster methods, Li et al. (2006) proposed the spiciness index parameter $(\beta)$, which is nondimensional and simplified from the definition given by Flament (2002). In their work, the boundaries of water masses were identified by the values of $\beta$; however, the salinity boundaries were prescribed a priori before measuring the temperature boundaries. 


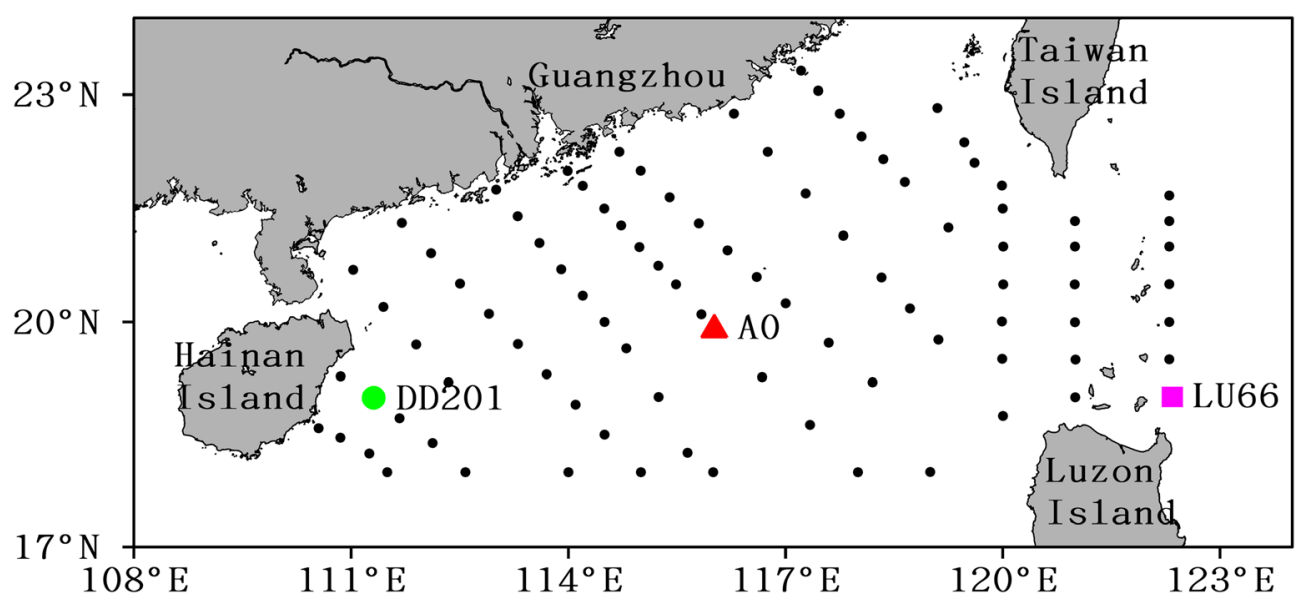

Figure 1. CTD stations (black dots) in the NSCS in the spring of 2011. The green circle, red triangle, and pink rectangle are three different stations to be compared in detail in the $\sigma-\pi$ diagram.

The South China Sea (SCS) is the largest semiclosed marginal sea in the northwest region of the Pacific Ocean. Due to the particularly complex water exchange between the northern SCS (NSCS) and the West Pacific Ocean (WPO), water masses in the SCS have been extensively studied. For instance, Fan et al. (1988) distinguished eight water masses in the NSCS with fuzzy cluster analysis. Li et al. (2002) classified the water masses from the bottom to the surface in the open SCS into six types with multimethods, including clustering, discriminant, and fuzzy analyses. Tian and Wei (2005) classified the water masses in the NSCS and Bashi Channel into four types with the T-S-based quadrangle method. Cheng et al. (2014) analyzed the water masses in the NSCS in summer using fuzzy cluster analysis and categorized the NSCS water in terms of five groups vertically.

However, the existing water mass clarification methods could not exactly identify the water mass center and boundary. Therefore, this work proposes a new curvilinear coordinate system for water mass analysis based on the potential density ( $\sigma$; sigma) and potential spicity/spiciness $(\pi ;$ pi) diagram. Note that the terminologies of spicity and spiciness are used by different authors with the same definition, in which the contours of spicity/spiciness are orthogonal to the contours of potential density. Despite extensive studies on potential spicity/spiciness (e.g., Huang, 2011; Huang et al., 2018; Jackett \& McDougall, 1985; McDougall \& Krzysik, 2015; Munk, 1981; Stommel, 1962), a clear way to define and calculate the potential spicity in practice remains debatable. Tailleux (2020) recently made an attempt to unify previous approaches to spiciness. The author showed that both "spiciness" and "spicity" can be regarded as variables orthogonal to density in the rescaled $\mathrm{X}(\mathrm{S})$ and $\mathrm{Y}(\theta)$ coordinates (here $\theta$ is potential temperature), where $\mathrm{X}(\mathrm{S})$ and $\mathrm{Y}(\theta)$ are nonlinear functions of $\mathrm{S}$ and $\theta$ for spiciness, while they are linear functions of $\mathrm{S}$ and $\theta$ for spicity.

The definition of potential spicity proposed by Huang et al. (2018) is chosen to do water mass analysis in this study, and the same terminology "spicity" as used by Huang et al. (2018) is also used in the following descriptions. The combination of potential spicity and potential density could provide a new tool in oceanography researches. In general, for large-scale dynamics, the vertical gradient of potential density controls the static stability, and the lateral gradient of density regulates the horizontal velocity through geostrophy. The motions of seawaters are primarily confined to quasi-adiabatic movements along the isopycnal surfaces. On the other hand, the potential spicity has no direct impact on large-scale dynamics. For mesoscale and submesoscale dynamics, the vertical gradient of spicity is a critically important parameter for double diffusion, while potential spicity may play an important role in modulating lateral mixing. However, the details of such roles remain unclear.

In this study, on the basis of the conductivity-temperature-depth (CTD) data observed in the NSCS from 30 April to 24 May 2011, we propose a $\sigma-\pi$ (or sigma-pi) diagram-based method to classify the water masses. With the help of this curvilinear coordinate system, the difficulty associated with identifying the boundaries between water masses can be easily solved by introducing a distance in the $\sigma-\pi$ space. Additionally, as an illustration example, we show that the intrusion of the WPO water into the NSCS 

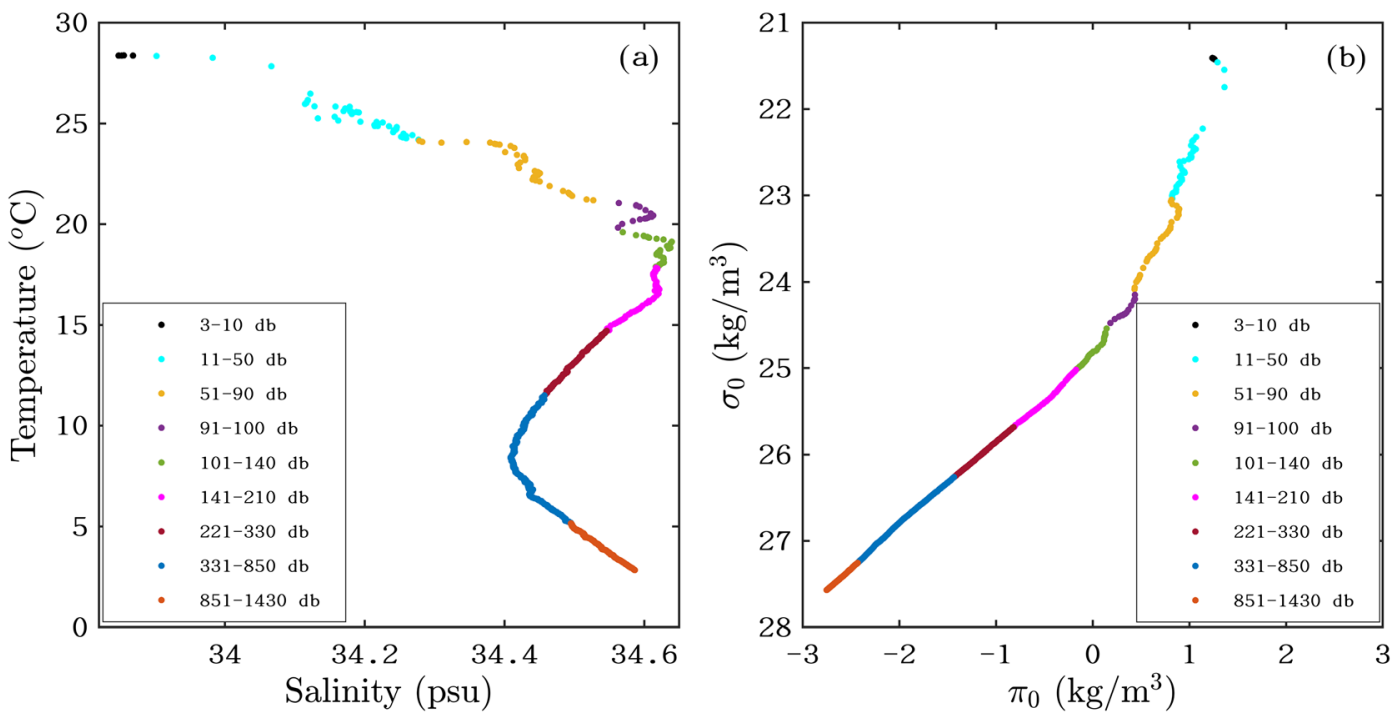

Figure 2. (a) T-S diagram and (b) $\sigma-\pi$ diagram for a single cast at a station (A0) in the NSCS.

can be clearly identified through this potential spicity analysis. Therefore, with the $\sigma-\pi$ coordinate system, seawater properties can be studied from an angle different from that of the traditional T-S diagram.

\section{Data and Method}

\subsection{Data}

In this study, our analysis is focused on the T and S data collected in the NSCS covering a region of $17-24^{\circ} \mathrm{N}$, $110-123^{\circ} \mathrm{E}$ (see Figure 1). Cruise observations were carried out onboard the R/V Dongfanghong 2 in the spring of 2011, using SBE911 or SBE917 plus CTD profiler manufactured by the Sea Bird Corporation. There are totally 92 stations, as shown in Figure 1. The vertical resolution of the CTD sampling is $1 \mathrm{~m}$.

\subsection{Data Preprocessing Method}

The potential spicity is calculated based on the UNESCO EOS_80 (Unesco, 1981, 1983) equation of state with parameters of in situ salinity $(s)$, in situ temperature $(t)$, pressure $(p)$, and reference pressure $\left(p_{r}\right)$, as described by Huang et al. (2018). The MATLAB code sw_pspi $\left(s, t, p, p_{r}\right)$, also provided by Huang et al. (2018), has been used to calculate the potential spicity (https://agupubs.onlinelibrary.wiley.com/action/downloadSupplement ?doi $=10.1029 \% 2 \mathrm{~F} 2018 \mathrm{JC} 014306 \% 26$ file $=2018 \mathrm{JC} 014306+$ Supporting+Information+Corrected.docx).

Figure 2 shows an example of the T-S diagram and corresponding $\sigma-\pi$ diagram based on the CTD data at a single station in the NSCS (A0; marked by a red triangle in Figure 1 ). Here, $\sigma_{0}$ and $\pi_{0}$ indicate the potential density and potential spicity based on a reference pressure of $p_{r}=0$ (the sea surface pressure). In this figure, the corresponding pressure ranges are illustrated in different colors. In Figure 2a, we empirically separate the T-S curve into nine segments, each of which may represent one water mass. As mentioned above, the boundaries of water masses are vaguely defined. In the upper layer (i.e., 0-200 dB), the temperature and salinity distributions seem rather irregular and appear as zigzag-like structures, which may correlate to the dynamical imprints of thermohaline intrusions and interleaving (see Figure 2a). On the other hand, the corresponding $\sigma-\pi$ curve has a much simpler feature and resembles a straight line, upon which examination reveals some fine prints of thermohaline intrusions in the upper part of the water column.

\section{Results}

\subsection{Potential Spicity and Potential Density Properties in the NSCS}

As shown in Figure 2, the seawater parameters measured by the CTD profiler can be used to calculate the potential density and potential spicity. Figure 3 displays all the $\sigma-\pi$ curves with the data collected from 


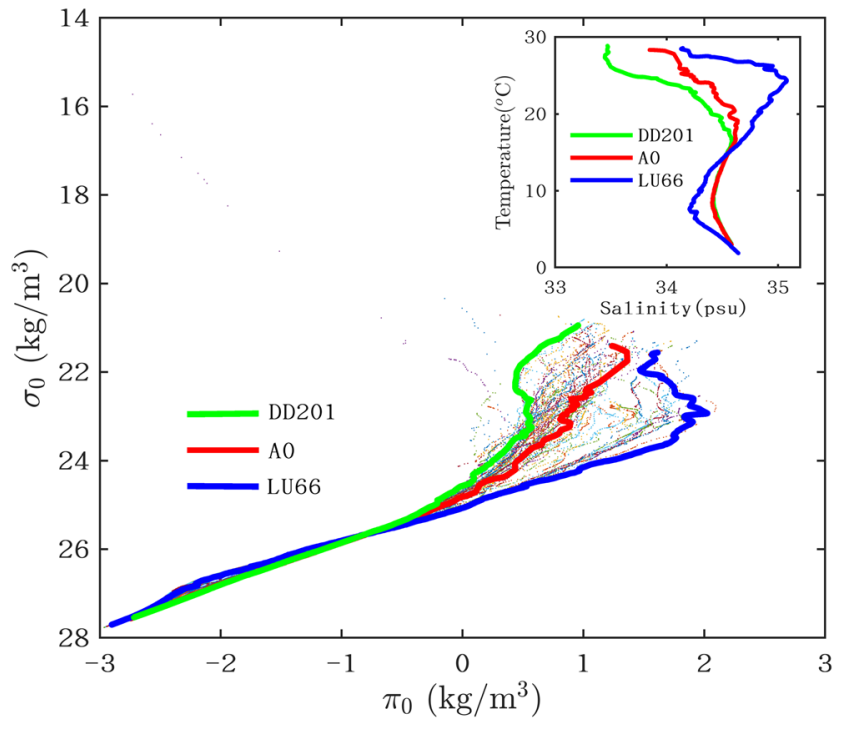

Figure 3. $\sigma-\pi$ curves for all CTD data collected during the 2011 spring cruise. The bold lines represent the $\sigma-\pi$ curves for three pivotal stations. Inset is the corresponding T-S curves of these three stations. the abovementioned cruise. The potential density and potential spicity data point fall into the vicinity of a single curve when the potential spicity value is lower than $-0.5 \mathrm{~kg} / \mathrm{m}^{3}$, and the corresponding potential density is around $25.7 \mathrm{~kg} / \mathrm{m}^{3}$. This isopycnal surface is the critical interface between the subsurface and intermediate layers. For the seawater with potential density smaller than $25.7 \mathrm{~kg} / \mathrm{m}^{3}$, the potential spicity is more dispersive. As potential density decreases, the water masses become spicier and the difference in spicity increases, corresponding to water masses located in the upper parts of the water column in the open ocean. In Figure 3, the green, red, and blue lines represent the $\sigma-\pi$ diagrams at three stations: in the west of NSCS (DD201), middle of NSCS (A0), and west of WPO (LU66) (see also Figure 1). The inset displays the corresponding T-S curves of these three stations. In the upper layer, the water masses observed at Station DD201 represent the typical characters of water masses in the NSCS, with relatively low temperature and low salinity features; while the water masses at Station LU66 are the typical WPO water masses, which hold high temperature and high salinity values; and those at Station A0 are the mixed product of water masses from the WPO and the NSCS. The red curve is located roughly halfway between the green and blue curves, indicating that in the upper layer, the water properties in potential spicity and potential density fields in the middle of the NSCS fall in between those in the WPO and the NSCS.

\subsection{Classifying Water Masses in Terms of Potential Spicity and Potential Density}

Water masses from different oceanic regions may have different properties, and their $\sigma-\pi$ curves could appear in different forms. Thus, before classifying the water masses, we first separate the $\sigma-\pi$ diagram into different parts. In the present case, we divide the diagram into two parts, one belonging to the NSCS and the other to the WPO. To clearly demonstrate the processes and results, we ignore the diluted water with small potential density in the following analysis.

First, we assume that the observation stations are uniformly distributed in the study region, and the NSCS is within a relatively narrow latitudinal band, so that we can ignore the latitude influence. That means the CTD observation at each grid point corresponds to a constant water volume. Here, we suppose each box is $100 \mathrm{~m} \times 100 \mathrm{~m}$ with a height of $1 \mathrm{~m}$. Then, the volume of each box is $10,000 \mathrm{~m}^{3}$. Using resolutions of $\Delta \pi_{0}=0.02 \mathrm{~kg} / \mathrm{m}^{3}$ and $\Delta \sigma_{0}=0.05 \mathrm{~kg} / \mathrm{m}^{3}, 276 \times 275$ grid boxes with the initial volumetric count being set as zero are generated on the $\sigma-\pi$ plane over the range of $\sigma_{0}=[14.0,27.8]\left(\mathrm{kg} / \mathrm{m}^{3}\right)$ and $\pi_{0}=[-3.2,2.3]$ $\left(\mathrm{kg} / \mathrm{m}^{3}\right)$. We begin with the water mass volumetric counting as follows. The potential spicity or potential density value of each box is checked, and then the corresponding grid in the $\sigma-\pi$ plane is found, and a volume of $10,000 \mathrm{~m}^{3}$ is added to that grid. In this way, the total water volume corresponding to various potential density or spicity value in the $\sigma-\pi$ plane is countered. Finally, the water volumes $\left(V_{i j}\right)$ in each grid in the $\sigma-\pi$ plane can be analyzed; the countered number in this volumetric census map represents a nominal volume, as shown in Figure 4a. Two major branches can be easily identified, suggesting two major water sources in our study region, that is, the NSCS and the WPO. To separate these water sources, we use a curve consisting of eight segments to trace a boundary defined by the smallest volumetric density and, thus, to divide the map into two parts. This curve is shown as a black line in Figure 4a. Using this boundary, the $\sigma-\pi$ diagram can be divided into two parts, as shown in Figure 4b, in which the green and red dots, respectively, indicate the water from the NSCS and the WPO.

Here, we choose the NSCS water as an example to illustrate the method of classifying water masses using potential density and potential spicity. Our discussion is focused on the potential density range of $21-28 \mathrm{~kg} / \mathrm{m}^{3}$. The classification of water masses consists of two parts: the identification of the center of each water mass and the corresponding boundary. Searching for the centers of water masses requires two steps. The first step is to identify the potential density of the water mass center, where the maximum and minimum values of potential spicity are selected as the range of spicity within each potential density layer. The second 


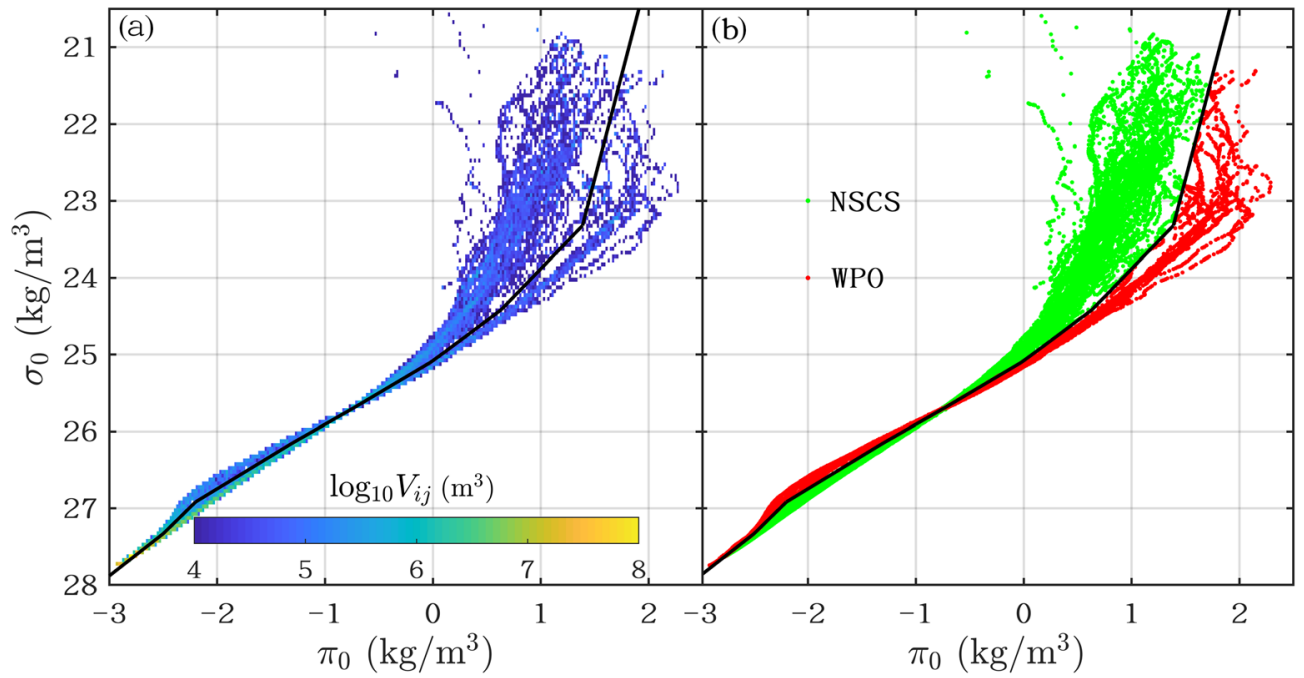

Figure 4. (a) Water volume $\left(\log _{10} V_{i j} ; \mathrm{m}^{3}\right)$ distribution in the $\sigma-\pi$ plane; (b) the green and red dots represent the water from the NSCS and the WPO, respectively. The black curve illustrates the boundary of two water sources.

step is to calculate the standard deviation (std) of potential spicity in each isopycnal layer, which can be used to quantify the variation and dispersion of the potential spicity. The results are displayed in Figure 5.

The solid curves in Figure 5 are the smoothed version of the range and std scatter points. They share a similar shape, especially when the potential density is larger than $25.7 \mathrm{~kg} / \mathrm{m}^{3}$. In the upper layer, the potential spicity varies over relatively wide ranges and big dispersions corresponding to the active environment in the upper ocean. The minimal values of these two curves (in the potential density range $21 \leq \sigma_{0} \leq 27 \mathrm{~kg} / \mathrm{m}^{3}$ ) are roughly located around $\sigma_{0}=25.73 \mathrm{~kg} / \mathrm{m}^{3}$ (see the black triangles in Figure 5). This means that there might be several water masses above and below this potential density layer. However, in the vicinal range of this layer, there is only one single type of water mass, which is called the SCS subsurface-intermediate mixed water (SUIMW). Similarly, all the troughs in the smoothed curves (marked by small triangles) may

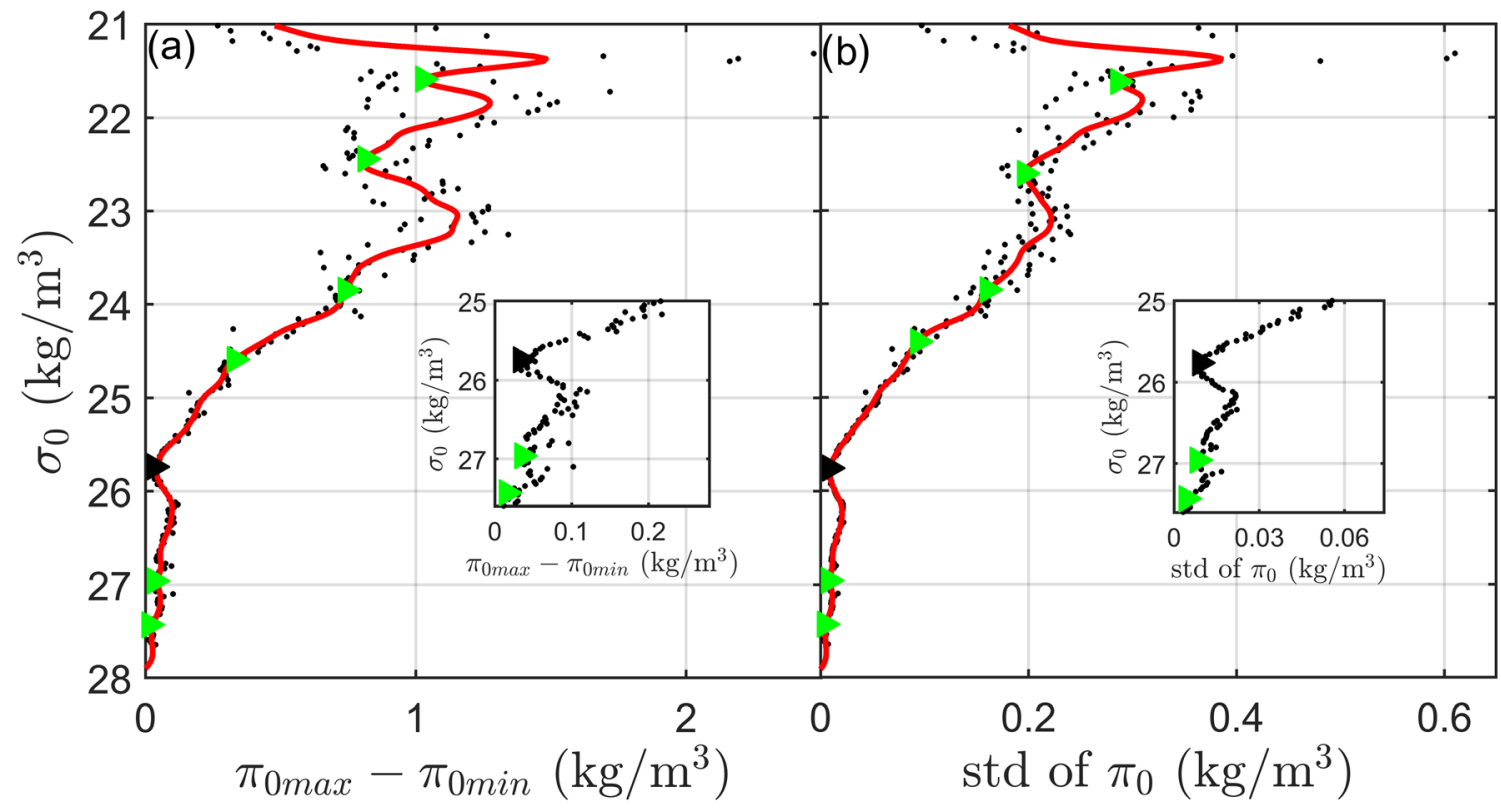

Figure 5. (a) The range of potential spicity for each potential density layer; (b) the standard deviation (std) of potential spicity for each potential density layer. The red curves are the smoothed version of the scatter points. The inserts are partial enlargements of the fine structures. 


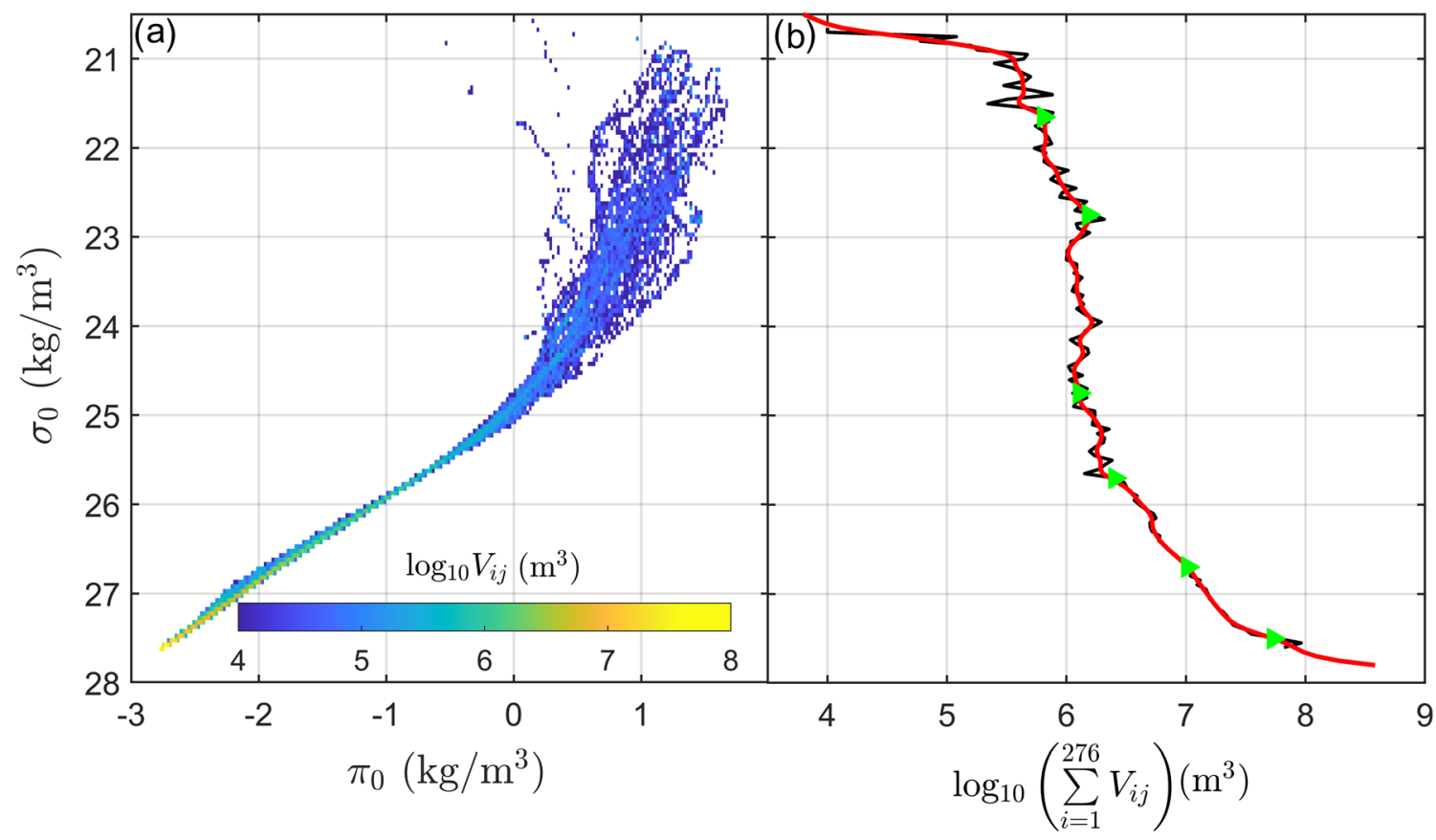
Figure 6. (a) NSCS water volume $\left(\log _{10} V_{i j} ; \mathrm{m}^{3}\right)$ distribution in the $\sigma-\pi$ plane; (b) total water mass volume $\left(\mathrm{m}^{3}\right)$ for each potential density interval, $\log _{10}\left(\sum_{i=1}^{276} V_{i j}\right)$.
The red curve is the smoothed version of the original water volumetric curve.

indicate the existence of a single water mass; while the peaks are clues to the transition of water mass types, which means that the corresponding potential density is between the potential densities of two different water masses. Therefore, from the potential spicity range and the std curves, some critical potential density layers can be distinguished. Note that these corresponding potential densities are suspected values instead of the confirmed potential densities of water mass centers. For identifying the real potential density values of water mass centers, we should also focus on the $\sigma-\pi$ plane-based NSCS water volumetric map (see Figure 6). Classifying water masses with volumetric diagram analysis was pioneered by Montgomery (1958) and subsequently reworked for the world oceans by Worthington (1981). Both Montgomery (1958) and Worthington (1981) used T-S plane to count the water volumes. In principle, volumetric diagram analysis is suitable for any set of properties, not just temperature and salinity, and can be produced and displayed (Talley et al., 2011). In this work, our analysis is based on potential density and potential spicity because their contours are practically orthogonal to each other, and they are in the same system of units, which could provide additional information in water mass analysis.

In general, the potential spicity and potential density of a water mass center can be identified from the grid with the volumetric maximum, as compared to the neighbor grids in the volumetric diagram. However, as Figure 6a shows, it is hard to directly detect the local maxima of water volume in this diagram. In fact, for many types of water masses, there is not necessarily a volumetric peak that is associated with a given water mass, which is particularly true for many water masses that are generated through mixing of different water masses. To illustrate the volume peaks more intuitively, we count the water volumes in each isopycnal layer and present the statistical results in a black curve in Figure 6b. Then, we smooth the original water volumetric curve to make the results more distinct (see the red curve in Figure 6b). Not all peaks in the curve are selected as the signs of the existence of water mass centers, due to the different numbers of sampling stations for each potential density layer. For instance, the peak occurring near $24 \mathrm{~kg} / \mathrm{m}^{3}$ is due to a sudden decrease in observation station numbers. Combined with the suspected potential density values obtained from Figure 5, the confirmed potential density values of water mass centers are identified in Figure 6b, which are marked as green triangles.

By volumetrically averaging potential spicity over the corresponding potential density layer in the water mass volumetric map, one can identify the center value of potential spicity for each water mass. The 

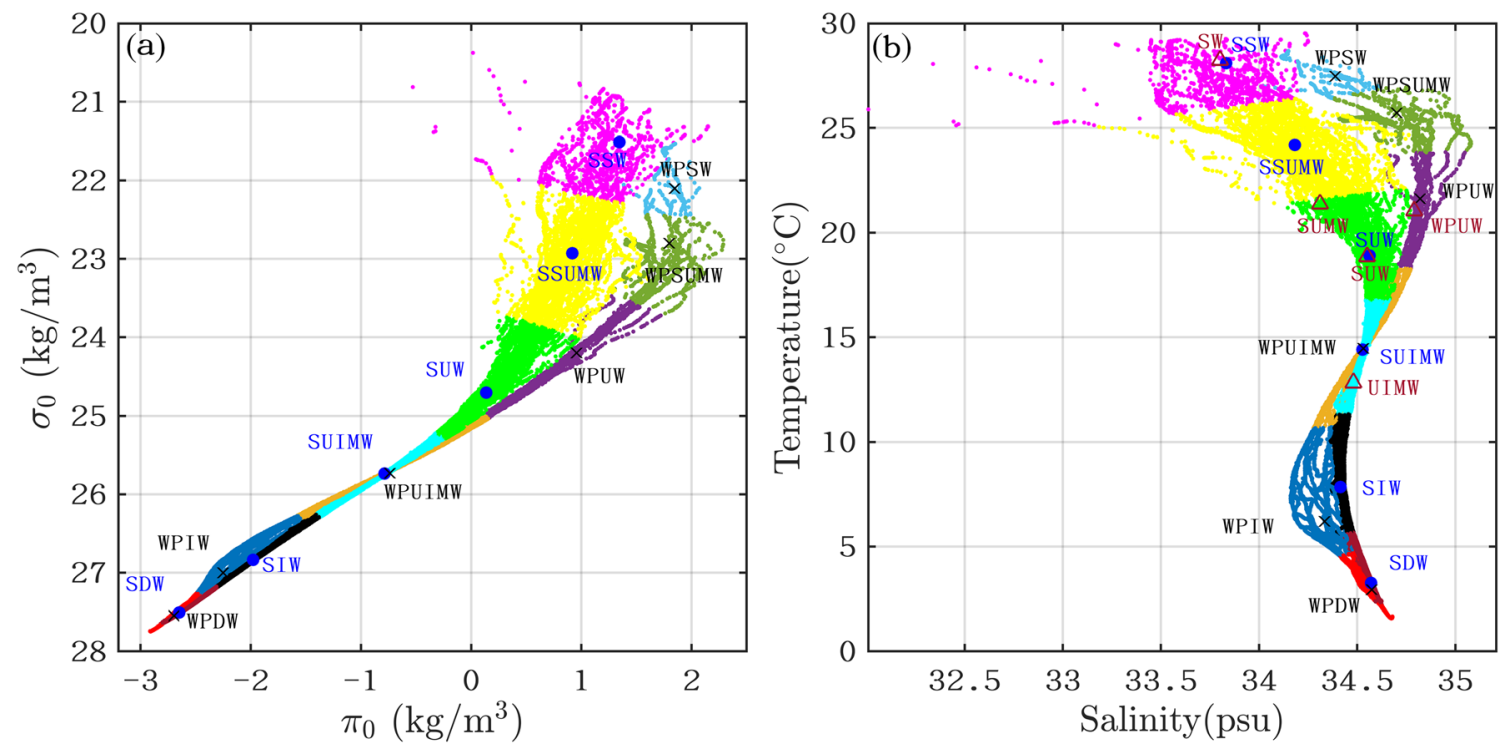

Figure 7. Water mass centers (a) in the $\sigma-\pi$ diagrams and (b) in the T-S diagrams are indicated by blue dots and black crosses, respectively. The small dots in the same color mean that they belong to the same water mass. The brown triangles in (b) indicate the water mass centers classified by the fuzzy cluster and TSSN analysis (from Zhu et al., 2019).

corresponding round-off values are used to define the water mass centers in terms of potential spicity, which are then used to define the potential density and potential spicity values of the water mass centers. Finally, seven water mass centers are distinguished in the NSCS. In a similar way, six other water mass centers are identified sourcing from the WPO. These centers correspond to the following water masses: the Zhujiang River diluted water (ZDW), SCS surface water (SSW), WPO surface water (WPSW), SCS surface-subsurface mixed water, WPO surface-subsurface mixed water (WPSUMW), SCS subsurface water (SUW), WPO subsurface water (WPUW), SUIMW, WPO subsurface-intermediate mixed water (WPUIMW), SCS intermediate water (SIW), WPO intermediate water (WPIW), SCS deep water (SDW), and WPO deep water (WPDW). The centers of the water masses sourcing from the NSCS and the WPO are illustrated as blue dots and black crosses, respectively, in the $\sigma-\pi$ diagrams, as shown in Figure 7a. To see the details clearly, the ZDW is also ignored in Figure 7.

The T-S values of the distinguished water mass centers can be retrieved from the corresponding water mass center values in the $\sigma-\pi$ space. Figure $7 \mathrm{~b}$ shows the water mass centers in the T-S diagram. Moreover, the water mass centers in both spaces and corresponding reference pressures are shown in Table 1.

Table 1

The Water Mass Centers in the $\sigma-\pi$ and T-S Spaces

\begin{tabular}{lrcrcr}
\hline Water mass & $\pi\left(\mathrm{kg} / \mathrm{m}^{3}\right)$ & $\sigma\left(\mathrm{kg} / \mathrm{m}^{3}\right)$ & $\mathrm{T}\left({ }^{\circ} \mathrm{C}\right)$ & $\mathrm{S}$ & $p_{r}(\mathrm{~dB})$ \\
\hline ZDW & -2.10 & 17.60 & 27.20 & 28.27 & 10 \\
SSW & 1.34 & 21.51 & 28.01 & 33.82 & 14 \\
SSUMW & 0.91 & 22.95 & 24.25 & 34.18 & 33 \\
SUW & 0.13 & 24.70 & 18.94 & 34.56 & 111 \\
SUIMW & -0.79 & 25.74 & 14.40 & 34.53 & 201 \\
SIW & -1.98 & 26.83 & 7.85 & 34.41 & 574 \\
SDW & -2.65 & 27.51 & 3.26 & 34.56 & 1,211 \\
WPSW & 1.85 & 22.10 & 27.47 & 34.39 & 13 \\
WPSUMW & 1.80 & 22.80 & 25.96 & 34.66 & 40 \\
WPUW & 0.95 & 24.20 & 21.63 & 34.82 & 156 \\
WPUIMW & -0.77 & 25.73 & 14.47 & 34.53 & 302 \\
WPIW & -2.26 & 27.00 & 6.20 & 34.33 & 706 \\
WPDW & -2.70 & 27.55 & 2.94 & 34.57 & 1,413 \\
\hline
\end{tabular}

Using the same CTD data set in this work, Zhu et al. (2019) utilized the fuzzy cluster and TSSN analysis to classify the water masses in the upper layer (above $300 \mathrm{~m}$ ) into six types: ZDW, surface water, surface-subsurface mixed water, SUW, WPUW, and subsurface-intermediate mixed water. These water mass centers are illustrated in Figure $7 \mathrm{~b}$ as brown triangles (the ZDW is also ignored). There are several differences and similarities between our results and those of Zhu et al. (2019). For example, in the surface layer, surface-subsurface mixed layer, and subsurface-intermediate mixed layer, the fuzzy cluster and TSSN analysis did not distinguish the water sourcing from the NSCS or from the WPO. On the other side, the centers of ZDW, WPUW, and SUW classified by these two methods are almost located in the same positions in the T-S diagrams. Further comparison with other studies, one can see that classifying the water masses using the $\sigma-\pi$ diagram is reasonable and robust. For example, Cheng et al. (2014) used the fuzzy cluster method to classify the water masses in the NSCS in the summer of 2011, in which the central values of temperature and 
salinity for the SIW are $8^{\circ} \mathrm{C}$ and 34.43, and those for the SDW are $3^{\circ} \mathrm{C}$ and 34.56 , respectively. These results are very close to ours. Zhang et al. (2004) analyzed the water masses in the source area of Kuroshio with fuzzy mathematics and distinguished five water masses in the WPO, that is, the WPSW, WPUW, WPUIMW, WPIW, and WPDW. Our results are in good agreement with theirs, except that we also classify the WPSUMW. In addition, the core temperature and salinity values of the other five WPO water masses are consistent with each other.

Compared to the water mass analysis based on the traditional T-S diagram, a superiority of using $\sigma-\pi$ diagram is that the contours of potential density and potential spicity are practically orthogonal. Additionally, the potential density and potential spicity are dimensionally homogenous, namely, they share a same unit system. Thus, the categories of water mass can be accurately judged by the measurement of a newly defined distance in the sigma-pi space. Consequently, the boundaries of water masses can be precisely detected by the distance between two water parcels in the $\sigma-\pi$ coordinate. The orthogonality and dimensional homogeneity of potential density and potential spicity can make the distance measurement in a precise and concise way, which is defined as below (Huang et al., 2018):

$$
d=\sqrt{\left(\sigma_{1}-\sigma_{2}\right)^{2}+\left(\pi_{1}-\pi_{2}\right)^{2}}
$$

where $\left(\sigma_{1}, \pi_{1}\right)$ and $\left(\sigma_{2}, \pi_{2}\right)$ are the potential density and potential spicity of these two water particles, respectively. With the distance measurement of water bodies, one can determine to which type of water mass the water particles belong by comparing the distances of particles to all the classified water mass centers. Evidently, the smaller the distance, the more similar the water properties. According to this criterion, different categories of water masses can be clearly distinguished in the $\sigma-\pi$ diagrams, as shown in Figure $7 \mathrm{a}$, in which the assembly of different colored dots represents different water masses and the boundaries of water masses are clearly defined between the distinct colored dots. Finally, the corresponding water mass classification results are displayed in the more intuitive T-S diagrams in Figure $7 \mathrm{~b}$. In comparison, if the traditional method based on the T-S diagram is used to classify the water mass, the boundaries of water masses can only be vaguely defined by experience. As for conventional cluster analysis, the boundaries are distinguished without any clear physical meanings and cannot be classified in some special regions. For instance, in the potential density range of $25.5 \leq \sigma_{0} \leq 27.5 \mathrm{~kg} / \mathrm{m}^{3}$, the WPO and the NSCS waters are incapable of separating using the fuzzy cluster method due to the similarity of temperature and salinity features in that range (Cheng et al., 2014). However, with the newly defined distance analysis in the $\sigma-\pi$ diagram, the boundaries of water masses can be easily distinguished, as Figure 7a shows.

\section{Discussion}

As shown above, the water in the study region is classified into 13 types with clearly defined boundaries in the $\sigma-\pi$ diagram. The $\sigma-\pi$-based method can be summarized as the following steps: (1) separate the water masses into two major categories, that is, the NSCS and the WPO, based on water volumetric distribution in the $\sigma-\pi$ plane; (2) find the potential densities of water mass centers according to the potential spicity range, std, and water volumetric curves; (3) identify the potential spicity of water mass centers by volumetrically averaging potential spicity within the corresponding potential density layer in the water mass volumetric map; and (4) classify the categories of water mass according to the distances of water parcels to all the water mass centers in the $\sigma-\pi$ space. Our results show the efficiency of using potential spicity to do water mass classification, though the physical meaning of either potential spicity or distance in the sigma-pi space remains to be further examined.

As mentioned above, the boundary of water sources is identified by the smallest volumetric density in the water volumetric map. Clearly, the boundary can be easily located above the potential density surface of $25.7 \mathrm{~kg} / \mathrm{m}^{3}$. Below this surface, the potential density and potential spicity features of seawater are relatively similar. The boundary below the $25.7 \mathrm{~kg} / \mathrm{m}^{3}$ isopycnal surface may be further refined; as a result, the classification of water masses below the intermediate layer may be refined in a future study.

In addition to using the $\sigma-\pi$ diagram to classify water mass, one can also use potential spicity to trace the evolution information of water masses if the data are available. As shown in Table 1, different water mass centers correspond to different potential spicity features and occupy specific isopycnal layers. Furthermore, as water 

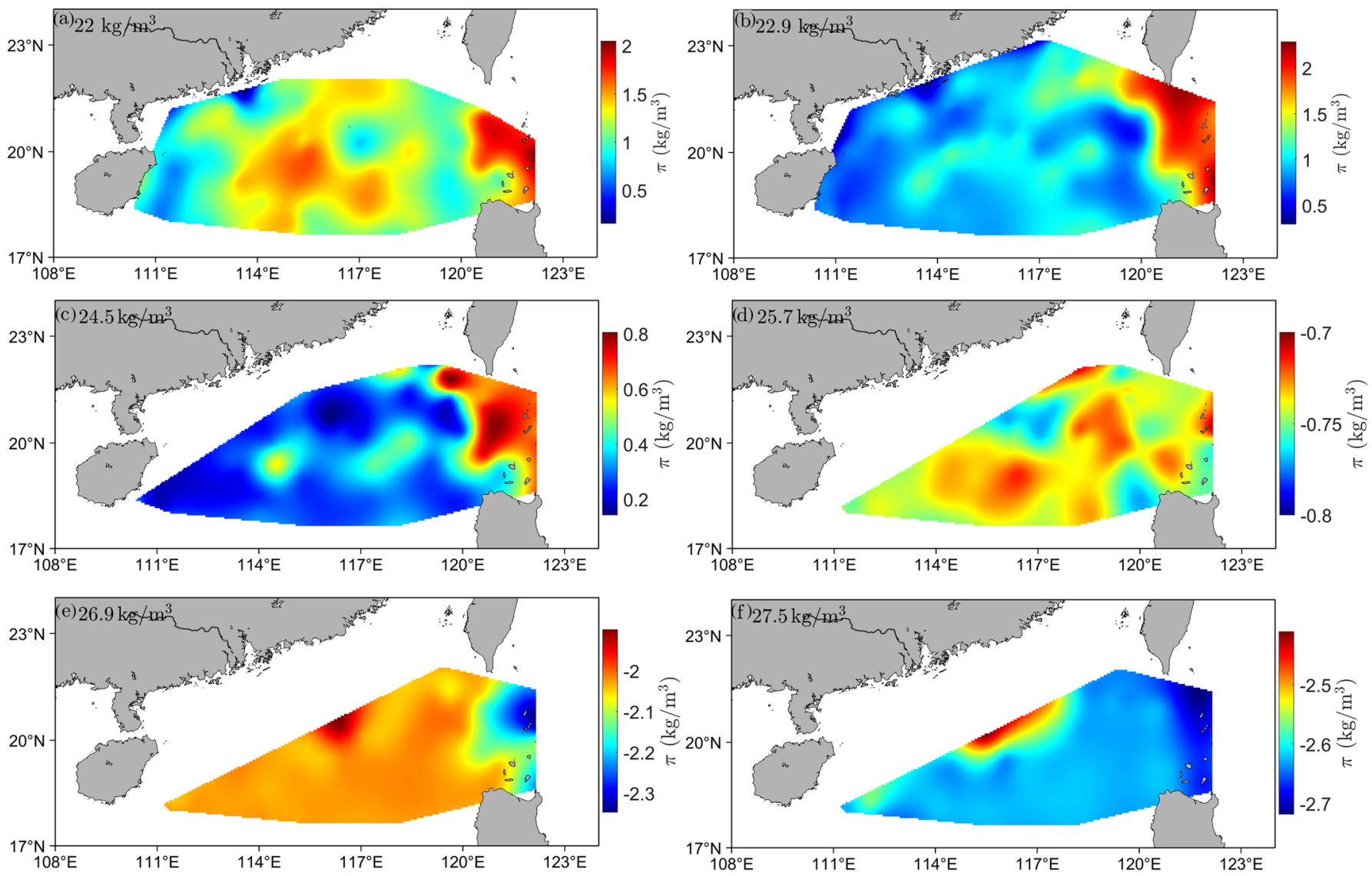

Figure 8. (a-f) The potential spicity distributions on various isopycnal surfaces. Isopycnal surface is denoted at the upper-left corner of each panel.

masses move primarily along the isopycnal surfaces, their physical characteristics, such as potential spicity, can gradually change. This means that potential spicity can be used as a tracer in studying the formation history of water masses. Herein, we select six representative isopycnal surfaces to analyze the water mass movement in the study region based on the spatial distribution of potential spicity. The results are shown in Figure 8. For the isopycnal surface of $22 \mathrm{~kg} / \mathrm{m}^{3}$, most areas of the NSCS are occupied by SSW and WPSW. Figures $8 \mathrm{a}, 8 \mathrm{~b}$, $8 \mathrm{c}$, and 8e show some tongue-like features associated with the WPO water extending into the NSCS via the north and middle of Luzon Strait, indicating the westward intrusion of the WPO water reaching about $119^{\circ} \mathrm{E}$ on the $24.5 \mathrm{~kg} / \mathrm{m}^{3}$ surface. These results are consistent with those of Li et al. (1998). Comparatively, on the surface of $25.7 \mathrm{~kg} / \mathrm{m}^{3}$ corresponding to the subsurface-intermediate layer, there are no clear intrusion features, revealing either homogeneous characteristics of water masses or a relatively weak east-west movement of water masses in this layer. Moreover, some high potential spicity patches can be detected in the central NSCS on the 22.9 and $24.5 \mathrm{~kg} / \mathrm{m}^{3}$ surfaces, which could be the signature of nonlinear Rossby eddies propagating into the NSCS through the Luzon Strait from the WPO (e.g., Cheng et al., 2014; Hu et al., 2012; Xie et al., 2016; Zheng et al., 2011, 2017; Zhu et al., 2019). The WPUW can traverse westward through the Luzon Strait and mix with the SUW, resulting in a subsequent decrease in its potential spicity, but it still has higher potential spicity than the local water. For the intermediate and deep layers, as shown in Figures 8e and 8f, the potential spicity in the NSCS is higher than that in the WPO, which corresponds to the relatively high temperature and high salinity features in the NSCS than those in the WPO on these two surfaces.

\section{Conclusion}

Using the $\sigma-\pi$ diagram, the distance between water parcels can be accurately calculated, and the water mass centers are defined in terms of the volume-weighted average. The boundaries between different water 
masses can be distinctly identified by calculating the newly defined distances of each water parcel to the water mass centers and then comparing these distances. Based on the ranges and std of potential spicity within each potential density layer and the $\sigma-\pi$ volumetric curve, the water masses in the NSCS in spring season are classified into 13 categories: ZDW, SSW, WPSW, SCS surface-subsurface mixed water, WPSUMW, SUW, WPUW, SUIMW, WPUIMW, SIW, WPIW, SDW, and WPDW. Compared with previous methods, including the fuzzy cluster and TSSN analysis, we can conclude that classifying water masses using the $\sigma-\pi$ diagram is reasonable and robust. Moreover, through the analysis of potential spicity distributions in some specific potential density layers, the formation history of water masses and propagations of waters can also be tracked. In the study region, the westward intrusion of the WPO water through the north and middle of Luzon Strait can be clearly identified based on the observation of tongue-like potential spicity structures. Also, some high spicity patches in the central NSCS may be due to the nonlinear Rossby eddies originating from the WPO and propagating into the NSCS. In conclusion, the $\sigma-\pi$ diagram proposed by Huang et al. (2018) is very useful for water mass analysis.

\section{Data Availability Statement}

Data used in this paper can be downloaded online (https://doi.org/10.6084/m9.figshare.11807577.v1).

\section{References}

This work was supported by the National Natural Science Foundation of China (91958203, 41776027, and 11732010) and the National Basic Research Program of China (2015CB954004 and 2009CB421208). Funding of Y.G.'s cotutelle doctoral research project by Région Hautsde-France and Xiamen University is acknowledged. All the cruise participants are appreciated. We also thank the editor and anonymous reviewers for their valuable comments.
Chen, S. J., \& Du, B. (1990). Water masses classification of the upper layer in the equatorial western Pacific using ISODATA of fuzzy cluster. Acta Oceanologica Sinica, 9(2), 187-201.

Cheng, G. S., Sun, J. D., Zu, T. T., Chen, J., \& Wang, D. X. (2014). Analysis of water masses in the northern South China Sea in summer 2011. Journal of Tropical Oceanography (in Chinese), 33(3), 10-16.

Fan, L. Q., Su, Y. S., \& Li, F. Q. (1988). Water mass analysis of the northern South China Sea. Acta Oceanologica Sinica (in Chinese), 10(2), 136-145.

Flament, P. (2002). A state variable for characterizing water masses and their diffusive stability: Spiciness. Progress in Oceanography, 54(1-4), 493-501. https://doi.org/10.1016/S0079-6611(02)00065-4

Hu, J. Y., Zheng, Q. A., Sun, Z. Y., \& Tai, C. K. (2012). Penetration of nonlinear Rossby eddies into South China Sea evidenced by cruise data. Journal of Geophysical Research, 117, C03010. https://doi.org/10.1029/2011JC007525

Huang, R. X. (2011). Defining the potential spicity. Journal of Marine Research, 69(4), 545-559. https://doi.org/10.1357/ 002224011799849390

Huang, R. X., Yu, L. S., \& Zhou, S. Q. (2018). New definition of potential spicity by the least square method. Journal of Geophysical Research: Oceans, 123, 7351-7365. https://doi.org/10.1029/2018JC014306

Jackett, D. R., \& McDougall, T. J. (1985). An oceanographic variable for the characterization of intrusions and water masses. Deep Sea Research Part A. Oceanographic Research Papers, 32(10), 1195-1207. https://doi.org/10.1016/0198-0149(85)90003-2

Jan, S., Tseng, Y. H., \& Dietrich, D. E. (2010). Sources of water in the Taiwan Strait. Journal of Oceanography, 66(2), 211-221. https://doi. org/10.1007/s10872-010-0019-7

Li, F. Q., Li, L., Wang, X. Q., \& Liu, C. L. (2002). Water masses in the South China Sea and water exchange between the Pacific and the South China Sea. Journal of Ocean University of Qingdao, 1(1), 19-24.

Li, F. Q., Xie, J., \& Li, Y. (2004). New methods of fitting the membership function of oceanic water masses. Journal of Ocean University of Qingdao, 3(1), 1-9. https://doi.org/10.1007/s11802-004-0001-3

Li, G. X., Han, X. B., Yue, S. H., Wen, G. Y., Yang, R. M., \& Kusky, T. M. (2006). Monthly variations of water masses in the East China Seas. Continental Shelf Research, 26(16), 1954-1970. https://doi.org/10.1016/j.csr.2006.06.008

Li, W., Li, L., \& Liu, Q. Y. (1998). Water mass analysis in the Luzon Strait and the northern South China Sea. Journal of Oceanography in Taiwan Strait (in Chinese), 17(2), 207-213.

McDougall, T. J., \& Krzysik, O. A. (2015). Spiciness. Journal of Marine Research, 73(5), 141-152. https://doi.org/10.1357/ 002224015816665589

Montgomery, R. B. (1958). Water characteristics of Atlantic Ocean and of world ocean. Deep Sea Research, 5(2-4), 134-148. https://doi.org/ 10.1016/0146-6313(58)90004-2

Munk, W. (1981). Internal waves and small-scale processes. In B. A. Warren \& C. Wunsch (Eds.), Evolution of physical oceanography (pp. 264-291). Cambridge: MIT Press.

Qiu, D. L., Zou, S. L., \& Li, C. M. (1984). A preliminary study on using cluster analysis method to classify the water masses in the Yellow Sea. Acta Oceanologica Sinica (in Chinese), 6(3), 281-292.

Stommel, H. (1962). On the cause of the temperature-salinity curve in the ocean. National Academy of Science, 48(5), 764-766. https://doi. org/10.1073/pnas.48.5.764

Su, Y. S., Yu, Z. X., \& Li, F. Q. (1989). Application of cluster analysis method for analyzing the water-masses in the shallow water area and the analysis of modified water masses in the Huanghai Sea and the East China Sea. Journal of Ocean University of Qingdao (in Chinese), 19(1), 71-85.

Tailleux, R. (2020). Spiciness theory revisited, with new views on neutral density, orthogonality and passiveness. Ocean Science Discussions. https://doi.org/10.5194/os-2020-39

Talley, L. D., Picard, G. L., Emery, W. J., \& Swift, J. H. (2011). Data analysis concepts and observational methods. In Descriptive physical oceanography: An introduction, (pp. 181-183). London, UL: Academic Press.

Thorpe, S. A. (2005). Convective instabilities. The turbulent ocean (pp. 115-143). New York: Cambridge University Press. https://doi.org/ 10.1017/CBO9780511819933 
Tian, T., \& Wei, H. (2005). Analysis of water masses in the northern South China Sea and Bashi Channel. Periodical of Ocean University of China (in Chinese), 35(1), 9-12.

Unesco (1981). The practical salinity scale 1978 and the international equation of state of seawater 1980. UNESCO Technical Papers in Marine Science, 36, 25.

Unesco (1983). Algorithms for computation of fundamental properties of seawater. UNESCO Technical Papers in Marine Science, 44, 53.

Worthington, L. V. (1981). The water masses of the world ocean: Some results of a fine-scale census. In Evolution of physical oceanography (pp. 42-69). Cambridge, MA: MIT Press.

Xie, L. L., Zheng, Q. A., Tian, J. W., Zhang, S. W., Feng, Y., \& Yi, X. F. (2016). Cruise observation of Rossby waves with finite wavelengths propagating from the Pacific to the South China Sea. Journal of Physical Oceanography, 46(10), 2897-2913. https://doi.org/10.1175/JPOD-16-0071.1

Zhang, X. D., Zhang, G. Y., Tong, K., \& Shen, H. (2004). Water mass analysis of the source area of Kuroshio. Marine Science Bulletin (in Chinese), 23(1), 15-21.

Zheng, Q. A., Tai, C. K., Hu, J. Y., Lin, H. Y., Zhang, R. H., Su, F. C., \& Yang, X. (2011). Satellite altimeter observations of nonlinear Rossby eddy-Kuroshio interaction at the Luzon Strait. Journal of Oceanography, 67(4), 365-376. https://doi.org/10.1007/s10872-011-0035-2

Zheng, Q. A., Xie, L. L., Zheng, Z. W., \& Hu, J. Y. (2017). Progress in research of mesoscale eddies in the South China Sea. Advances in Marine Science (in Chinese), 35(2), 131-158.

Zhu, J., Zheng, Q. A., Hu, J. Y., Lin, H. Y., Chen, D. W., Chen, Z. Z., et al. (2019). Classification and 3-D distribution of upper layer water masses in the northern South China Sea. Acta Oceanologica Sinica, 38(4), 126-135. https://doi.org/10.1007/s13131-019-1418-2 